\title{
ESTUDIO DE LA RESISTENCIA A FLEXO-COMPRESIÓN DE ELEMENTOS NO ESBELTOS DE FÁBRICA DE BLOQUES DE HORMIGÓN, CON Y SIN ARMADURA, A PARTIR DEL ENSAYO DE MURETES*
}

\author{
(AN INVESTIGATION INTO THE FLEXO-COMPRESSIVE STRENGTH OF NON SLENDER \\ CONCRETE MASONRY WALLS, WITH AND WITHOUT REINFORCEMENT, BY TESTING \\ SMALL MASONRY SPECIMENS)
}

L. Villegas y N. Tuesta, Drs. Ingenieros de Caminos

J. Ibáñez, Ingeniero de Caminos

Departamento de Diseño y Construcción de Estructuras.

Fecha de recepción: 19 - XII - 94

ETS de Ingenieros de Caminos de Santander-Universidad de Cantabria.

ESPAÑA

RESUMEN

En este artículo se presenta una confrontación teóricoexperimental del comportamiento resistente de secciones de fábrica, a partir del elemento de ensayo (murete) propuesto por el Eurocódigo EC-6[6].

Para llevar a cabo esta investigación se han ensayado 39 muretes, con y sin armadura, así como los materiales básicos de los mismos; estudiándose, además, la influencia que tiene en su resistencia el efecto de la saturación.

\section{SUMMARY}

This article presents a theoretical-experimental confrontation of the resistance behavior of masonry cross-sections, through the element of research (small specimens) proposed by Eurocódigo EC-6[6].

In order to carry out this investigation 39 small specimens (with and without reinforcement), as well as their basic materials, have been tested; the influence that the effect of saturation has in the strength of the masonry elements has also been analyzed.

\section{Alcance de la investigación}

\subsection{Objetivos}

Los objetivos fundamentales, que corresponden a este artículo, son los siguientes:

- Obtener experimentalmente, a partir del elemento de ensayo (murete) propuesto por el Eurocódigo EC-6, la resistencia a compresión de las fábricas en estudio. Estos resultados se confrontarán con los que se obtienen, analíticamente, a partir de la resistencia de los materiales constituyentes: las de la pieza y el mortero en las fábricas simples, más la del homigón de relleno en las fábricas armadas.
- Investigar experimentalmente el comportamiento de "muretes" de $1 \mathrm{~m}$ de altura y $(0,14 \mathrm{x}$ $\left.1,00 \mathrm{~m}^{2}\right)$ de sección transversal, simples y armados, bajo cargas excéntricas. Estos resultados se confrontarán con los que suministran diferentes teorías, de uso habitual, sobre el comportamiento resistente de "secciones de fábrica" solicitadas por flexo-compresiones transversales a su plano.

- Adicionalmente se evaluará, experimentalmente, las influencias que tienen el hormigón relleno y la saturación en la resistencia de los muretes ensayados.

\footnotetext{
* Este artículo forma parte de la Tesis Doctoral [10] realizada por el Ing. Tuesta y dirigida por el Prof. Villegas, habiendo obtenido la calificación de Apto Cum Laude.
} 


\subsection{Modelo experimental seleccionado}

El modelo experimental elegido es un esquema de excentricidades de carga iguales y del mismo sentido (véase figura 1).

\subsection{Plan de ensayos}

A fin de cumplir con los objetivos de esta investigación, en su parte experimental se ha programado el plan de ensayos de la tabla 1.

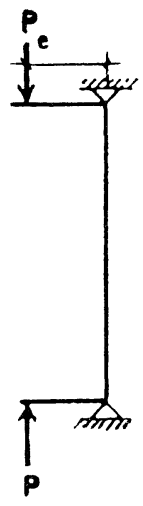

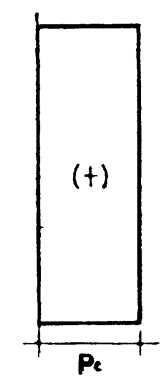

(a)

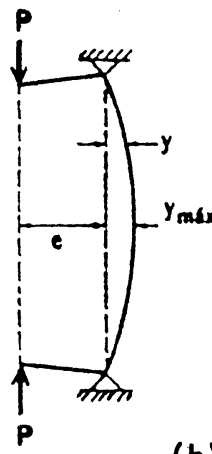

(b)

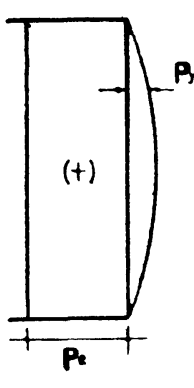

Fig. 1. Esquema de excentricidades elegido. Momentos existentes $\sin ($ a) y con (b) efecto de esbeltez.

\section{Tabla 1}

Plan de ensayos

\begin{tabular}{|l|l|}
\hline \multicolumn{1}{|c|}{ Ensayos } & \multicolumn{1}{|c|}{ Variables investigadas } \\
\hline Materiales constituỵentes & $\begin{array}{l}\text { Todas (Bloques, Morteros, } \\
\text { Hormigón de relleno y Arma- } \\
\text { duras). }\end{array}$ \\
\hline $\begin{array}{l}\text { Muretes } \\
\left.\text { (De } 1 \times 1 \mathrm{~m}^{2}\right): 39 \text { elementos. } \\
\text { (*) Espesor del bloque, } \\
\mathrm{t}=14 \mathrm{~cm} .\end{array}$ & $\begin{array}{l}\text { - Influencia del hormigón } \\
\text { de relleno. } \\
\text { - Influencia de la saturación. }\end{array}$ \\
\hline
\end{tabular}

\section{Resistencia a compresión de la fábrica a partir del ensayo de muretes}

El obtener la resistencia a compresión de una fábrica, a partir del ensayo de muretes, es una propuesta que corresponde al EC-6. El ensayo, según CEN/TC 125/N63 [5], se realiza sobre un mínimo de 3 muretes, estableciéndose sus dimensiones a partir de la tabla 2. En la figura 2 se muestra un elemento de ensayo para el caso de $1=21_{u}$.
Tabla 2

Dimensiones de los muretes [5]

\begin{tabular}{|c|c|c|c|c|c|}
\hline \multicolumn{2}{|c|}{$\begin{array}{c}\text { Dimensiones de } \\
\text { la unidad }\end{array}$} & \multicolumn{3}{|c|}{$\begin{array}{c}\text { Dimensiones de los muretes para el } \\
\text { ensayo de compresión de la fábrica }\end{array}$} \\
\hline $1_{u}(\mathrm{~mm})$ & $\mathrm{h}_{\mathrm{u}}(\mathrm{mm})$ & Longitud (l) & \multicolumn{2}{|c|}{ Altura (h) } & Espesor (t) \\
\hline$\leq 300$ & $\begin{array}{l}\leq 150 \\
>150\end{array}$ & $1 \geq 2 \mathrm{l}_{\mathrm{u}}$ & $\begin{array}{l}\mathrm{h} \geq 5 \mathrm{~h}_{\mathrm{u}} \\
\mathrm{h} \geq 3 \mathrm{~h}_{\mathrm{u}}\end{array}$ & $3 \mathrm{t} \leq \mathrm{h} \leq 15 \mathrm{t}$ & $\mathrm{t}=\mathrm{t}_{\mathrm{u}}$ \\
\hline$>300$ & $\begin{array}{l}\leq 150 \\
>150\end{array}$ & $1 \geq 1.5 \mathrm{l}_{\mathrm{u}}$ & $\begin{array}{l}\mathrm{h} \geq 5 \mathrm{~h}_{\mathrm{u}} \\
\mathrm{h} \geq 3 \mathrm{~h}_{\mathrm{u}}\end{array}$ & $\mathrm{h} \geq \mathrm{l}$ & \\
\hline
\end{tabular}
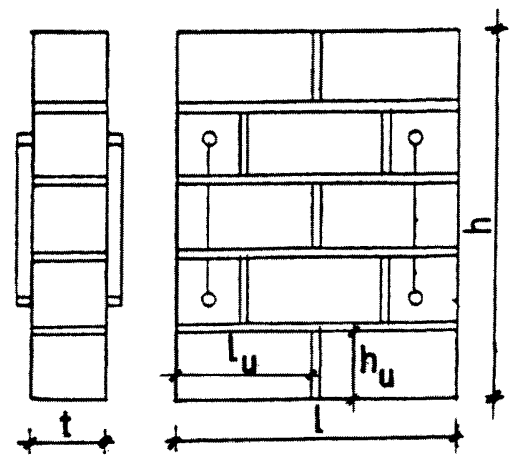

Fig. 2. Murete de ensayo segúncen [5].

\section{Análisis resistente de la sección, sometida a flexo-compresión, en el estado límite de agotamiento}

El análisis a realizar depende de que la sección de la fábrica tenga o no armadura.

\subsection{Análisis resistente de secciones no armadas}

Dentro de los métodos de cálculo de secciones de fábrica, propuestos por normativas e investigaciones, se contemplan cuatro de ellos:

\subsubsection{Método del bloque uniforme de presiones} propuesto por las normativas EC-6 [6], PIET 70 [8] y B.S. 5628 [3]

Estas normas consideran que para el caso de una sección maciza, bajo una carga exterior, se puede suponer que en el estado límite de agotamiento la sección responderá con un bloque rectangular de presiones, tal como se muestra en la figura 3. Para el caso de fábricas de bloques huecos la B.S. [3] propone que puede asumirse, conservadoramente y a efectos de diseño, que las unidades se comporten como sólidas, siempre que se refiera la resistencia característica a compresión de la pieza a su área bruta. 


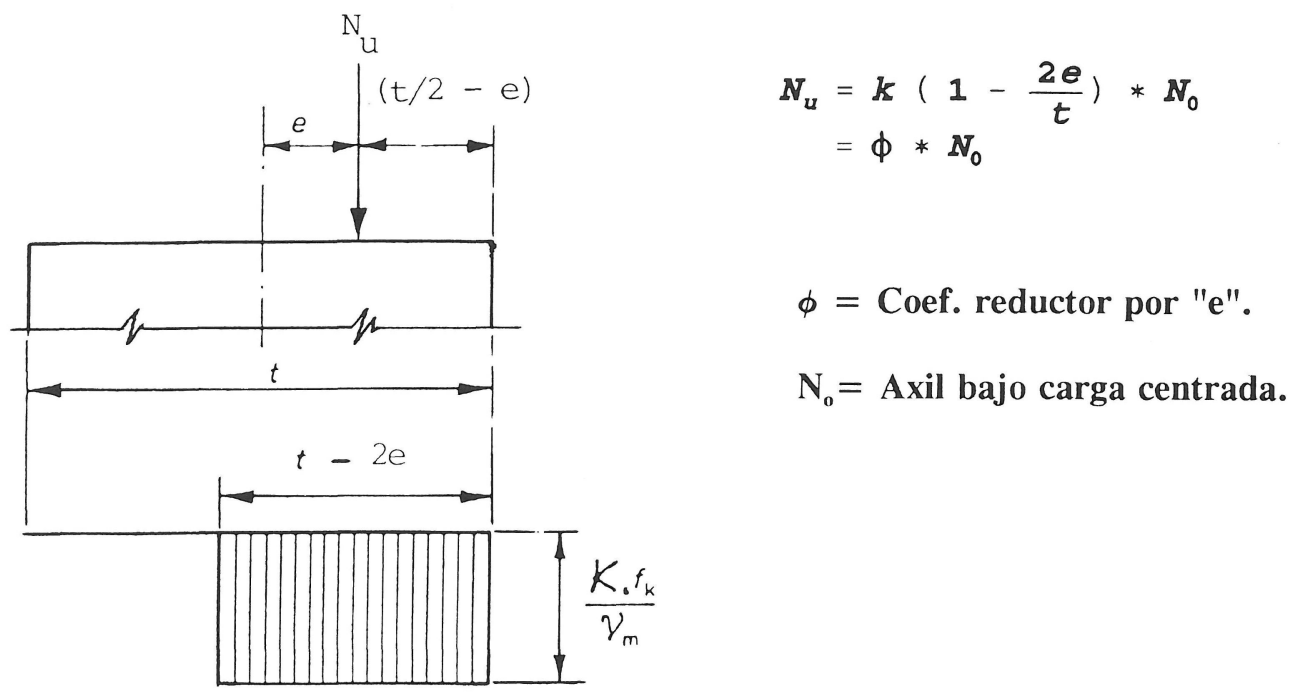

Fig. 3. Esquema del método del bloque uniforme de presiones.
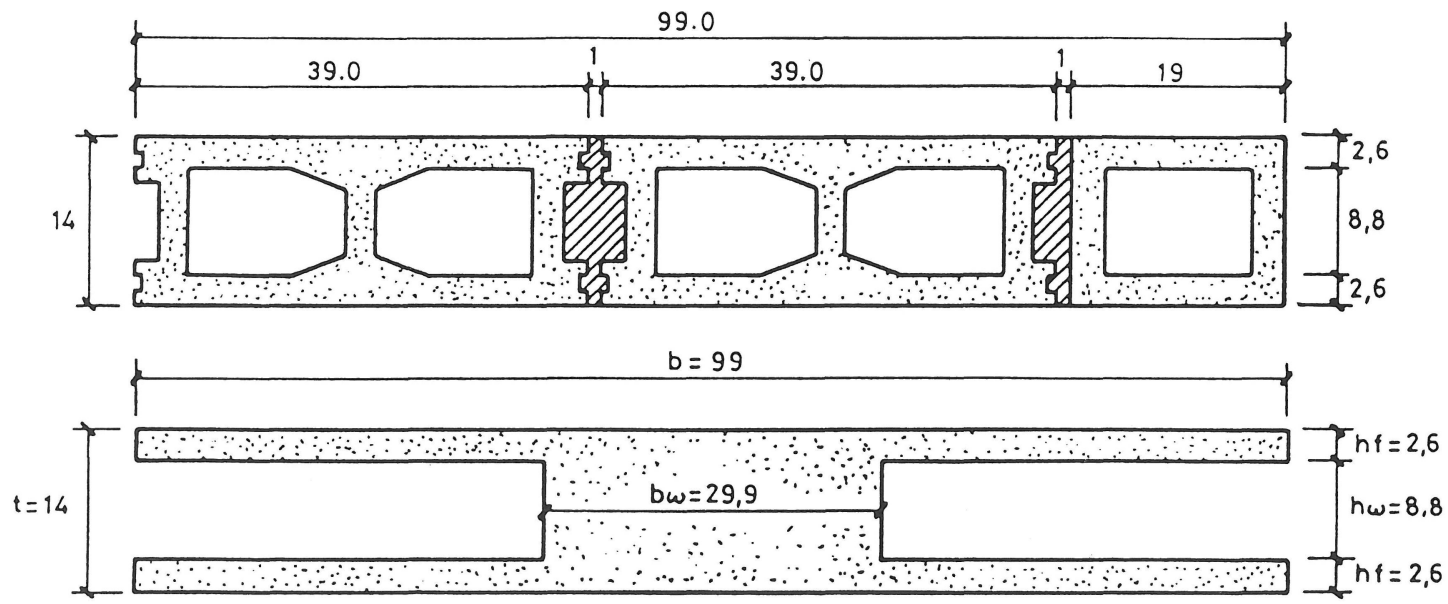

Fig. 4. Secciones, original y equivalente, para el método del bloque uniforme de presiones en secciones "I" (BUPSI)

\subsubsection{Método del bloque uniforme de presiones en} secciones "I" (BUPSI) [10]

Este método es la adaptación del análisis que se realiza en secciones "I", al caso de secciones de fábricas de bloques buecos, para lo cual se concentran los espesores de los tabiques transversales de la fábrica, tal como se muestra en la figura 4. Se admite bloque uniforme de presiones en aquella parte de la sección cuyo baricentro coincide con el punto de aplicación de la carga excéntrica.

\subsubsection{Método del bloque triangular de presiones propuesto por F. Yokel [13]}

Para analizar la sección de una fábrica sin armar, independientemente de que sea bueca o maciza,
Yokel estableció una alternativa de cálculo basada en un comportamiento lineal de la fábrica hasta la rotura. Los diagramas de tensiones que se generan en la sección de la fábrica, para las distintas excentricidades de la carga, se muestran en la figura 5.

\subsubsection{Método general de análisis de secciones armadas}

Para el caso de una cuantía de acero igual a cero, este método (ver 3.2) es aplicable a secciones no armadas. En esta investigación se ha utilizado el programa desarrolado por L. Villegas [12], al que se ha denominado programa general de cálculo de fábricas $(P G C F)$. 


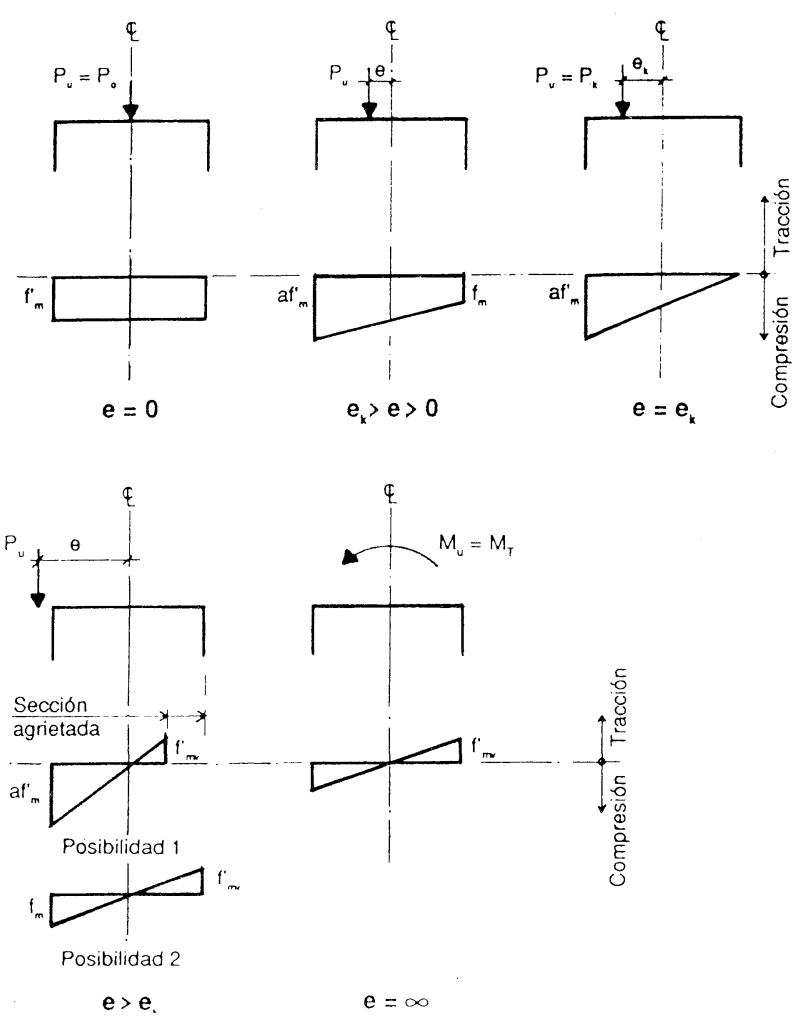

Fig. 5. Tensiones de rotura propuesto por F. Yokel [13], para diferentes excentricidades de carga.
En la figura 6 se muestran los dominios de deformación de una sección armada, para el caso particular en que la armadura se dispone en el centro de las piezas.

\section{Investigación experimental realizada}

\subsection{Propiedades de los materiales básicos}

Las propiedades de los materiales básicos vienen indicadas en la figura 7 y tablas 3 a 8 .

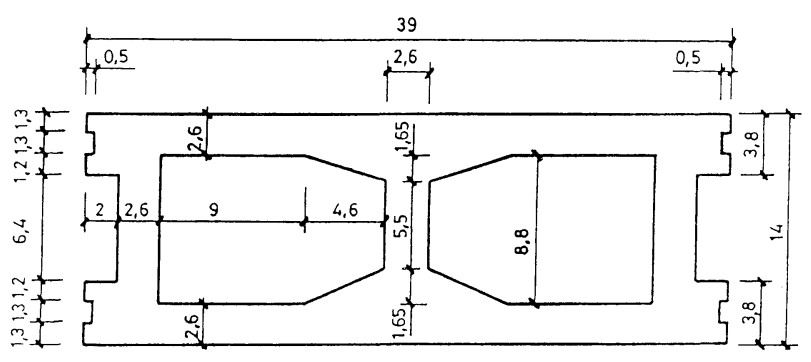

Fig. 7. Dimensiones del bloque utilizado en la investigación.

Tabla 3

\section{Resistencia a compresión de bloques saturados ensayados según UNE 41-172 [11]}

\begin{tabular}{|c|c|c|c|c|}
\hline \multirow{2}{*}{ Fase } & \multirow{2}{*}{$\begin{array}{c}\text { Edad } \\
\text { (días) }\end{array}$} & $\begin{array}{c}\text { Carga de } \\
\text { rotura } \\
\text { media }(\mathrm{Mp})\end{array}$ & \multicolumn{2}{|c|}{$\begin{array}{r}\text { Resistencia med. a compresión } \\
\left(\mathrm{kp} / \mathrm{cm}^{2}\right)\end{array}$} \\
\cline { 4 - 5 } & & S/ secc. bruta & S/ secc. neta \\
\hline II & 342 & 55,3 & 101,3 & 188,3 \\
III & 61 & 44,5 & 81,5 & 151,6 \\
\hline
\end{tabular}

Nota: Área bruta $=546 \mathrm{~cm}^{2}$

Área neta $=293,62 \mathrm{~cm}^{2}$

(valores según geometría)

Fig. 6. Dominios de deformación de la sección de un muro de fábrica armada.

\subsection{Análisis resistente de secciones armadas}

El análisis resistente de fábricas armadas es similar al que se realiza en las secciones de hormigón armado. Las hipótesis básicas y los dominios de deformación de la sección vienen definidas en el EC-6 [7] y BS [4]. En esta investigación, para el análisis resistente de la sección, se ha utilizado el programa $P G C F[12]$ antes mencionado.

Tabla 4

\section{Dosificación del mortero utilizado}

\begin{tabular}{|c|c|c|c|c|}
\hline \multirow{2}{*}{ Fase } & \multirow{2}{*}{$\begin{array}{c}\text { Dosificación } \\
(\mathrm{M}-80)\end{array}$} & \multirow{2}{*}{$\begin{array}{c}\text { Relación } \\
\text { agua/cemento }\end{array}$} & \multicolumn{2}{|c|}{ Consistencia media } \\
\hline & & & Cono de Abrams & Mesa de sacudida \\
\hline II & Cemento $=1$ & $1,10-1,20$ & 18 & 123,5 \\
\hline III & $\begin{array}{l}\mathrm{Cal}=0,5 \\
\text { Arena }=4\end{array}$ & $1,20-1,28$ & 18,2 & \\
\hline
\end{tabular}


Tabla 5

Relación entre las resistencias a compresión de probetas de mortero, elaboradas en moldes absorbentes e impermeables

\begin{tabular}{|c|c|c|c|c|c|c|c|c|c|}
\hline \multirow{3}{*}{ Fase } & \multicolumn{6}{|c|}{$\begin{array}{l}\text { Resistencia media a compresión } \\
\qquad\left(\mathrm{kp} / \mathrm{cm}^{2}\right)\end{array}$} & \multirow{2}{*}{\multicolumn{3}{|c|}{$\begin{array}{c}\text { Relación } \\
\text { M. absorb / M. imp. }\end{array}$}} \\
\hline & \multicolumn{3}{|c|}{ Prob. mold. imperm. } & \multicolumn{3}{|c|}{ Prob. mold. absorb. } & & & \\
\hline & 7 & 28 & 90 & 7 & 28 & 90 & 7 & 28 & 90 \\
\hline II & 136,3 & 172 & 175,7 & 166,6 & $\begin{array}{l}254,7 \\
\text { (1) }\end{array}$ & 270,7 & 1,22 & 1,48 & 1,54 \\
\hline III & 101,3 & 144,8 & 171 & 146,9 & $\begin{array}{l}179,8 \\
(2)\end{array}$ & 188,7 & 1,45 & 1,24 & 1,10 \\
\hline
\end{tabular}

Observaciones (mortero y hormigón de relleno):

(1) Probetas curadas por saturación.

(2) Probetas curadas en sus moldes a condiciones naturales y saturadas durante 48 horas antes del ensayo.

Tabla 7

Relación entre las resistencias a compresión de probetas de hormigón de relleno, elaboradas en moldes absorbentes e impermeables

\begin{tabular}{|c|c|c|c|c|c|c|c|c|c|}
\hline \multirow{3}{*}{ Fase } & \multicolumn{6}{|c|}{$\begin{array}{l}\text { Resistencia media a compresión } \\
\qquad\left(\mathrm{kp} / \mathrm{cm}^{2}\right)\end{array}$} & \multirow{2}{*}{\multicolumn{3}{|c|}{$\begin{array}{c}\text { Relación } \\
\text { M. absorb / M. imp. }\end{array}$}} \\
\hline & \multicolumn{3}{|c|}{$\begin{array}{l}\text { Prob. mold. imperm. } \\
\qquad(\varnothing 15, \mathrm{~h}=30)\end{array}$} & \multicolumn{3}{|c|}{$\begin{array}{l}\text { Prob. mold. absorb. } \\
\qquad(7 \times 7 \times 14 \mathrm{~cm})\end{array}$} & & & \\
\hline & 7 & 28 & 90 & 7 & 28 & 90 & 7 & 28 & 90 \\
\hline II & 173,7 & 234,7 & 252,3 & 212,2 & $\begin{array}{l}335,6 \\
\text { (1) }\end{array}$ & 383,8 & 1,22 & 1,43 & 1,52 \\
\hline III & 184 & 241,7 & 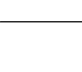 & 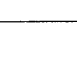 & $\begin{array}{l}325,5 \\
\text { (2) }\end{array}$ & 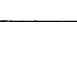 & & 1,34 & \\
\hline
\end{tabular}

Tabla 6

\section{Dosificación del hormigón de relleno utilizado}

\begin{tabular}{|c|c|c|c|}
\hline Fase & Dosificación & $\begin{array}{c}\text { Relación } \\
\text { agua/cemento }\end{array}$ & $\begin{array}{l}\text { Consistencia } \\
\text { media }(\mathrm{cm})\end{array}$ \\
\hline II & \multirow{2}{*}{$\begin{array}{l}\text { Cemento=1 } \\
\text { Arena=2,25 } \\
\text { Arido grueso=1 }\end{array}$} & $0,74-0,75$ & 18,3 \\
\hline III & & $0,80-0,83$ & 18,3 \\
\hline
\end{tabular}

Tabla 8

\section{Características geométricas de la armadura} de junta

\begin{tabular}{|c|c|c|c|c|c|}
\hline Tipo & $\begin{array}{c}\text { Ancho } \\
(\mathrm{mm})\end{array}$ & $\begin{array}{c}\text { Diámetro } \\
\text { alambre } \\
\text { longitudinal } \\
(\mathrm{mm})\end{array}$ & $\begin{array}{c}\text { Diámetro } \\
\text { alambre } \\
\text { diagonal } \\
(\mathrm{mm})\end{array}$ & $\begin{array}{c}\text { Dist. entre } \\
\text { soldaduras } \\
(\mathrm{mm})\end{array}$ & $\begin{array}{c}\text { Sección de 2 } \\
\text { alambres } \\
\text { longitudinales } \\
\left(\mathrm{mm}^{2}\right)\end{array}$ \\
\hline RND, $^{\prime} \mathrm{Z}$ & 100 & 4 & 3,75 & 406 & 25 \\
\hline
\end{tabular}

La distribución de la armadura en los muretes armados ha sido tanto vertical como horizontal. En cuanto a la armadura vertical, se ha optado por utilizar un acero de $10 \mathrm{~mm}$ de diámetro por cada alveolo del bloque. Este acero, distribuido a lo largo del eje del murete, conduce a una cuantía geométrica del 0,28 por ciento; mayor en un 40 por ciento del mínimo establecido en las normas ( 0,2 por ciento). El tipo de acero ha sido AEH-400S, con un límite elástico nominal de $5.576,3 \mathrm{kp} / \mathrm{cm}^{2}$.

En lo que respecta a la armadura horizontal, se ha colocado una celosía (tipo Murfor RND) en cada junta horizontal del mortero. El diámetro elegido ha sido de $4 \mathrm{~mm}$, obteniéndose así una cuantía geométrica de 0,07 por ciento (mínima establecida por las normas). 


\subsection{Especimenes de investigación}

Para la longitud del bloque elegido (39 cm), el CEN [5] especifica el uso de muretes de longitud y altura mayor o igual que $58,5 \mathrm{~cm}$. En esta investigación se ha optado por usar muretes, simples y armados, de $\left(1,0 \times 1,0 \mathrm{~m}^{2}\right)$.

\subsubsection{Construcción de muretes}

La primara hilada de los muretes ha sido construida dentro de un perfil metálico, dispuesto para facilitar el transporte hasta el pórtico de carga, que luego constituiría el cabezal inferior de apoyo en el ensayo. Los bloques de la primera hilada, tanto en los muretes simples y armados, se asentaron encima de una capa de mortero de $1 \mathrm{~cm}$ de espesor, que fue esparcida en toda la superficie del perfil.

El transporte de los muretes, desde el área de construcción hasta el pórtico de carga, ha sido concebido para que todo su peso sea tomado desde su zona inferior; evitándose así el daño de las juntas (véase figura 11).

\subsubsection{Construcción de muretes simples}

Los muretes simples han sido construidos en base a cinco hiladas, cada una de ellas formada por dos bloques enteros y un medio bloque; siendo el espesor de junta, horizontal y vertical, de $1 \mathrm{~cm}$. En lo que respecta a la colocación del mortero, éste ha sido dispuesto para lograr un llenado completo de las juntas verticales, así como su distribución en toda la sección transversal neta del murete (full mortar bedding).

Para el refrentado de los muretes se han tenido que preparar "bloques ciegos", a fin de poder esparcir uniformemente la capa de mortero (véase figura 8). El curado de estos especímenes se realizó, protegiéndolos con un plástico, durante sus primeros siete días.

\subsubsection{Construcción de los muretes armados}

Estos muretes, con la armadura establecida en el apartado 4.1, fueron construidos de tal manera que su primera y última hilada se formaron con bloques del tipo "dintel"; esto ha permitido el anclaje por soldadura de las varillas en el perfil de asiento (véase figura 9), y un doblado de $25 \mathrm{~cm}$ en su extremo superior.

Construida la primera fila de bloques, se procedió al vaciado del hormigón de relleno; continuándose

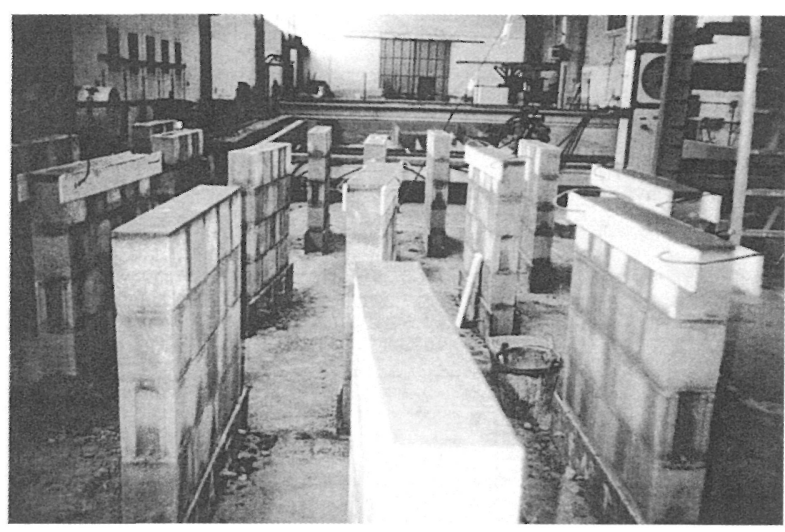

Fig. 8. Refrentado de muretes simples.

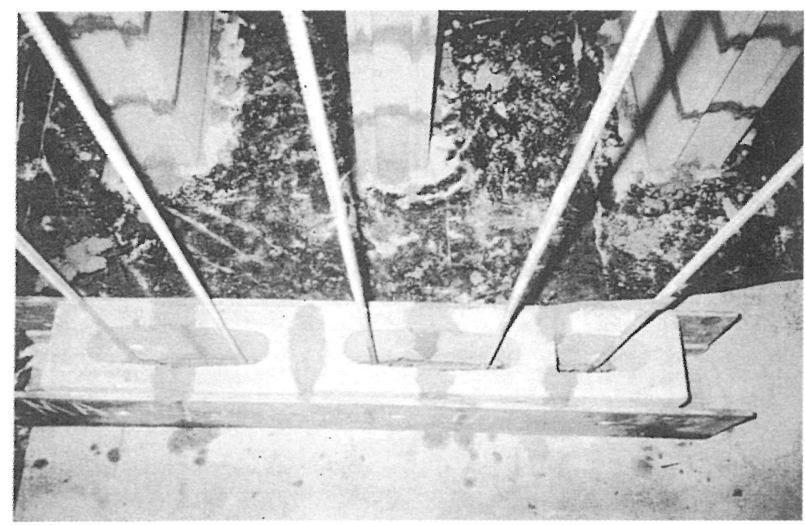

Fig. 9. Disposición de los bloques dinteles, en la primera bilada de los muretes armados.

con el asentado de las piezas hasta la penúltima hilada. Pasadas las 24 horas se rellenaron éstas y se asentó la última hilada procediéndose, una vez dobladas las varillas, a su vaciado. La construcción por etapas facilitó el relleno completo.

Cabe destacar que durante el asentado de bloques se utilizaron esponjas, a fin de impedir que el mortero sobrante quedase atrapado dentro del murete; asimismo la compactación del hormigón de relleno se realizó en forma manual, mediante picado con una varilla de $10 \mathrm{~mm}$ de diámetro. En la figura 10 se esquematiza, en un murete armado, la distribución que adopta el hormigón de relleno.

\subsubsection{Ensayo de muretes}

Para el transporte de los muretes se dispuso de una "celda metálica", concebida para que todo su peso fuese tomado desde su zona inferior (evitándose así el daño de las juntas) y cuyas características geométricas se muestran en la figura 11. Los especímenes de ensayo fueron acercados al pórtico de carga mediante un puente grúa. 


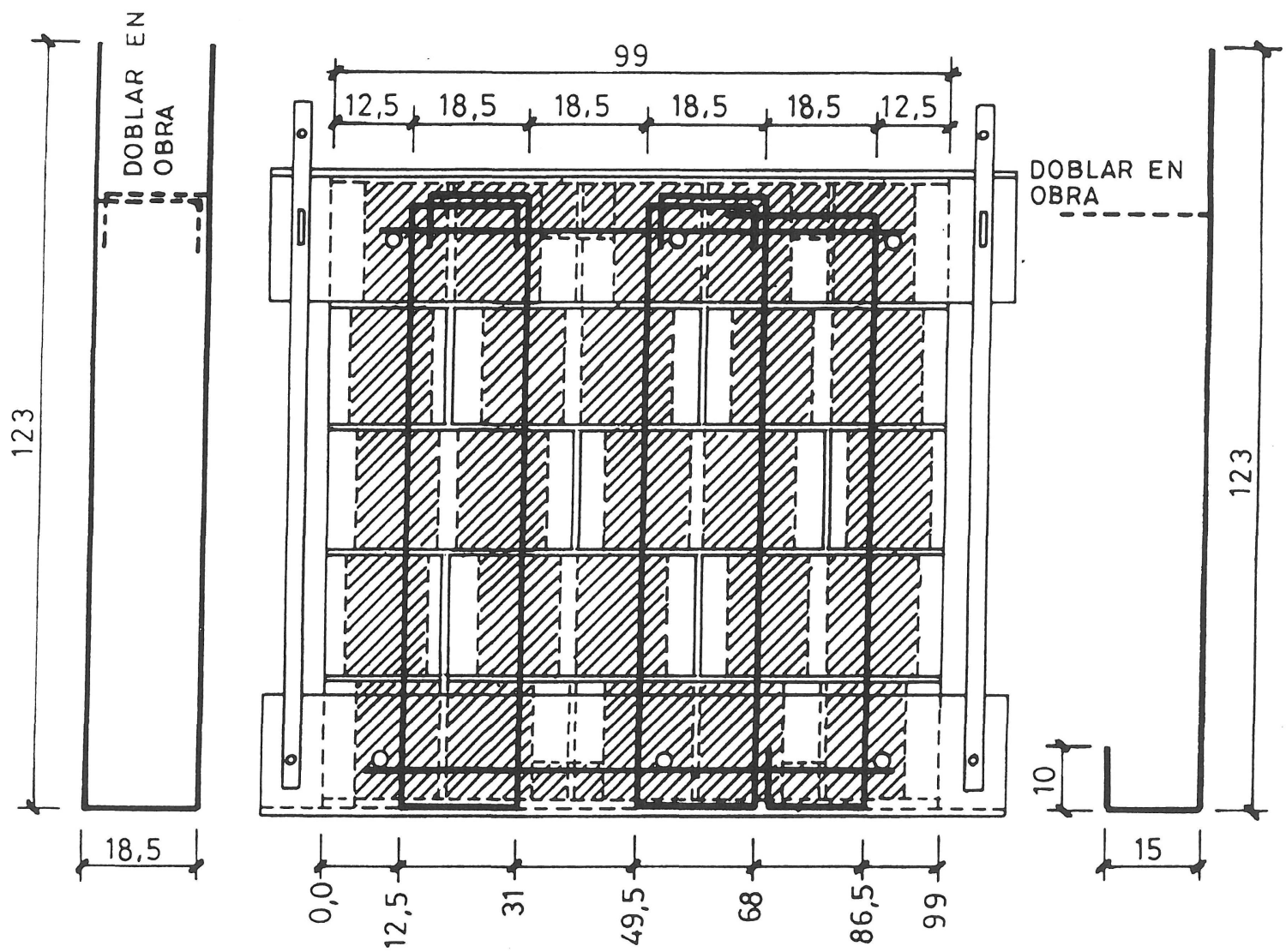

Fig. 10. Distribución del hormigón de relleno en murete armado.
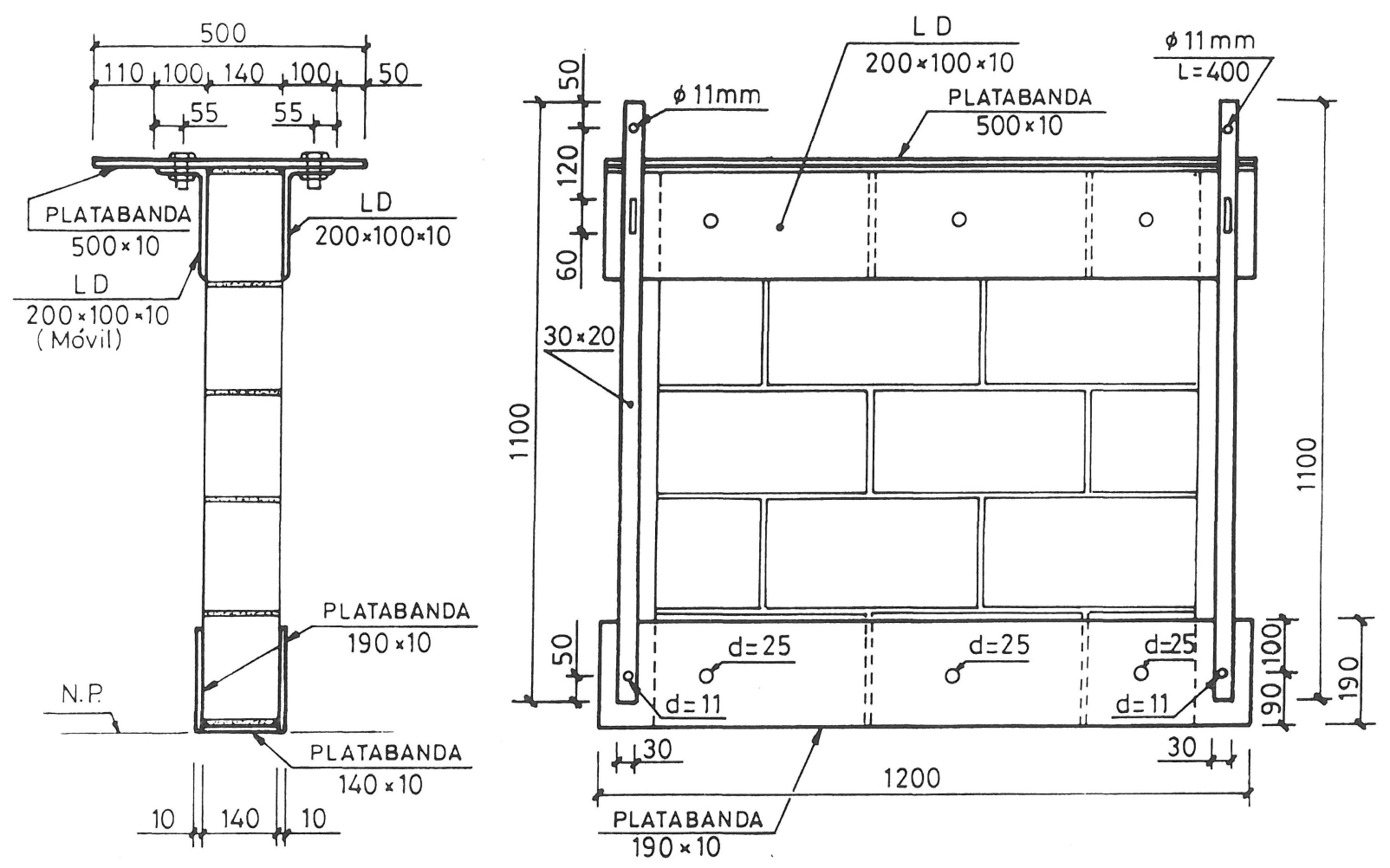

Fig. 11. Celda metálica para transporte de muretes. 


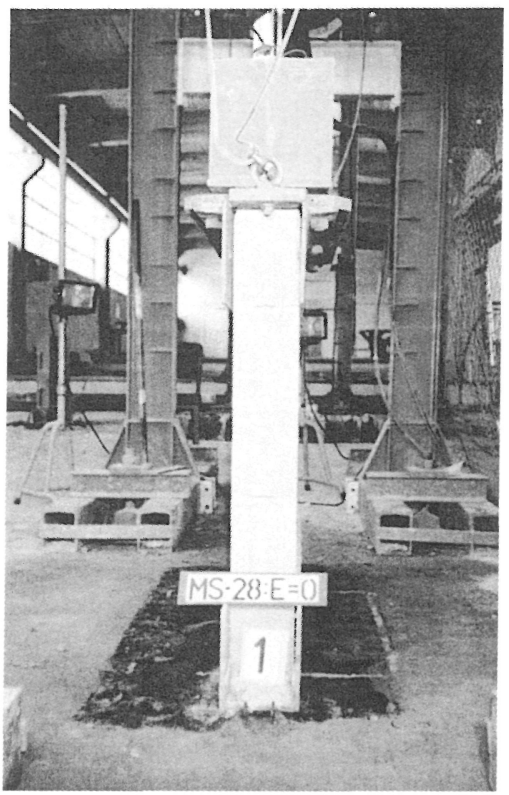

Fig. 12. Esquema de carga centrada en murete simple.

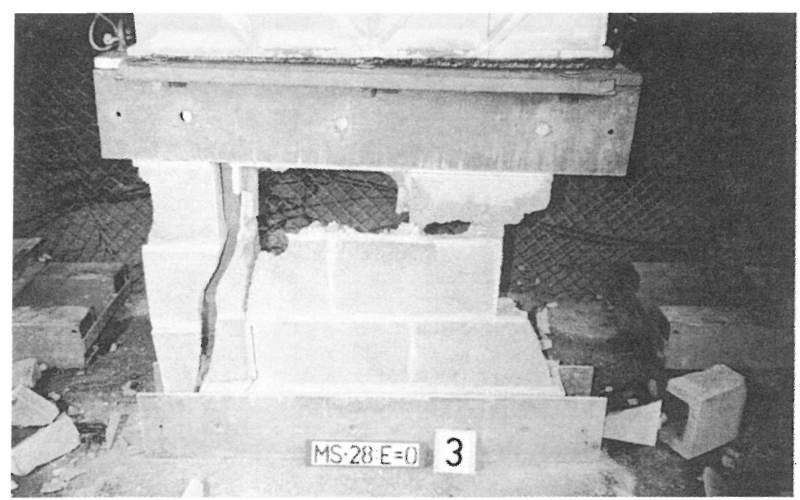

Fig. 13. Rotura de murete simple a carga centrada.

Para evitar la separación de los angulares del cabezal superior de la "celda metálica", durante el ensayo de muretes armados, se optó por unir dichos angulares (con pernos de alta resistencia) al espécimen de ensayo.

En los muretes que tenían que ser ensayados a carga centrada, ésta ha sido aplicada uniformemente en los cabezales. Para el ensayo de los elementos a carga excéntrica, la transmisión de carga fue a través de rodillos de $48 \mathrm{~mm}$ de diámetro. El movimiento transversal de los muretes, durante el ensayo, fue impedido por unos accesorios que sirvieron para establecer las diferentes excentricidades.

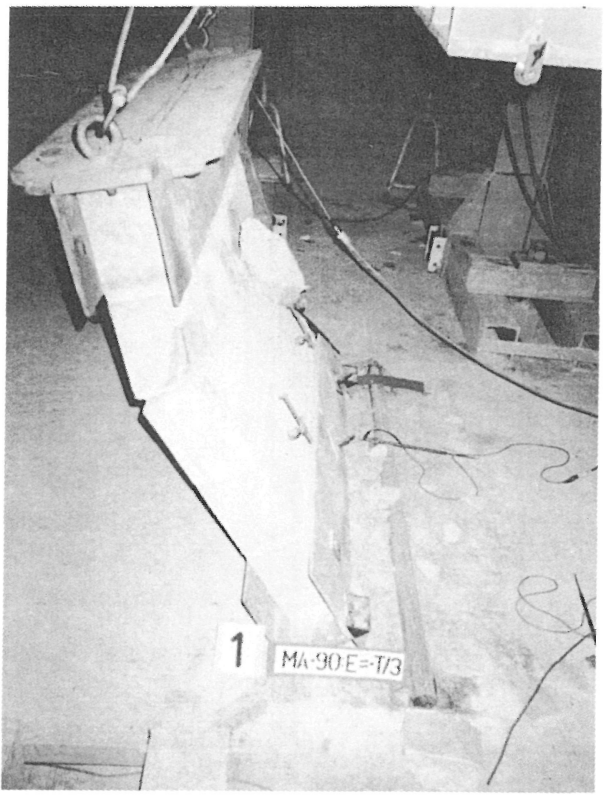

Fig. 14. Aspecto de la zona comprimida, en rotura, de murete armado $(e=t / 3)$

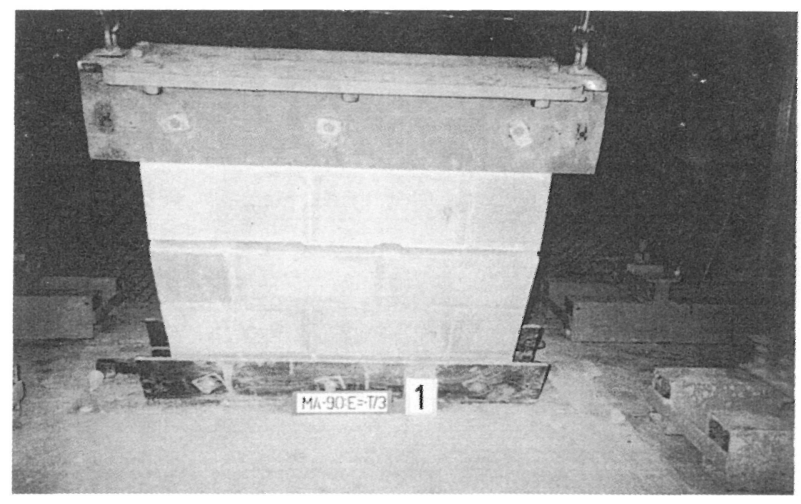

Fig. 15. Aspecto de la zona traccionada, en rotura, de murete armado $(e=t / 3)$

Los muretes fueron ensayados de tal manera que la carga de rotura, esperada para cada elemento, fue aplicada entre 15 a 30 minutos (según norma CEN/TC 125, número 63 [5]).

En los ensayos de muretes simples a carga centrada, figuras 12 y 13 , se ha podido observar que éstos desarrollan, antes de su rotura, fisuras verticales en sus caras laterales. Estas fisuras aparecen por las tensiones de tracción que desarrolla el mortero en los bloques. Debe indicarse que, en base a la disponibilidad de carga $(160 \mathrm{Mp}$ ) del Laboratorio de Estructuras, no fue posible ensayar muretes armados a carga centrada. 
Los especímenes que tenían que ser ensayados en forma saturada, fueron sumergidos totalmente en una piscina con agua, con un tiempo de saturación de 48 horas para los muretes simples y 72 horas para los armados.

En lo que respecta a los muretes ensayados a carga excéntrica, la falla se produjo por aplastamiento de la fábrica en su zona comprimida. En las figuras 14 y 15 se muestra el aspecto de la rotura típica, en muretes armados sometidos a cargas excéntricas.

\section{Análisis de los resultados de muretes. Confrontación teórico-experimental}

\subsection{Muretes simples sometidos a carga centrada}

En la tabla 9 se relacionan las resistencias experimentales a compresión de muretes simples secos, característica y media, con las obtenidas a partir de las normas analizadas. Puede observarse, de la relación "EXP/TEO", que las normas analizadas con una pequeña diferencia en el EC-6, dan una buena aproximación con respecto a la resistencia a compresión experimental de los muretes simples ensayados.

Tabla 9

Relación entre las resistencias a compresión experimental y teórica en muretes secos (fase III)

\begin{tabular}{|l|c|c|c|}
\hline $\begin{array}{l}\text { Propuesta de } \\
\text { cálculo }\end{array}$ & $\begin{array}{c}\text { Resistencia } \\
\text { experimental } \\
\left(\mathrm{kp} / \mathrm{cm}^{2}\right)\end{array}$ & $\begin{array}{c}\text { Resistencia } \\
\text { teórica } \\
\left(\mathrm{kp} / \mathrm{cm}^{2}\right)\end{array}$ & $\begin{array}{c}\text { Relación } \\
\text { "Exp. teo." }\end{array}$ \\
\hline $\begin{array}{l}\text { EC-6: } \\
\mathrm{F}_{\mathrm{K}}=0,55 \mathrm{~F}_{\mathrm{b}}^{0,65 *} \mathrm{~F}_{\mathrm{m}}^{0,25} \\
\text { (Res. caract. en secc. bruta) }\end{array}$ & 49,4 & 54,4 & 0,91 \\
\hline $\begin{array}{l}\text { BS: } \\
\text { Tablas } \\
\text { (Res. caract. en secc. bruta) }\end{array}$ & 49,4 & 37,6 & 1,31 \\
\hline $\begin{array}{l}\text { NCMA: } \\
\text { Tablas } \\
\text { (Res. media en secc. efect.) }\end{array}$ & 110,7 & 99,2 & 1,12 \\
\hline $\begin{array}{l}\text { ACI: } \\
\text { Tablas } \\
\text { (Res. media en secc. efect.) }\end{array}$ & 110,7 & 99,8 & 1,11 \\
\hline
\end{tabular}

(*) Sección bruta $=99 \times 14 \mathrm{~cm}^{2}$.

Sección efectiva $=\sum$ Áreas netas de bloques.
5.1.1 Coeficientes de paso "murete/prisma simples", para carga centrada

El por qué de esta relación radica en que a partir de la resistencia de prismas [2], que son mucho más fáciles de construir y de ensayar que los muretes, puede llegarse a predecir las cargas de rotura de la fábrica.

El coeficiente de paso "murete/prisma simples", determinado experimentalmente en la fase III, es cuantificado en la tabla 10. Los valores encontrados han sido: 0,72 cuando la evaluación se hace sobre el área efectiva o bruta de los elementos de ensayo, y 0,66 cuando se evalúa sobre su área neta (área efectiva más área rellena con mortero).

Tabla 10

\section{Coeficiente de paso, en resistencia media a compresión, entre prismas simples de tres unidades (P3) y muretes simples (mS)}

\begin{tabular}{|l|c|c|c|}
\hline \multirow{2}{*}{$\begin{array}{l}\text { Tipo de } \\
\text { area } \\
\text { transversal }\end{array}$} & \multicolumn{2}{|c|}{$\begin{array}{c}\text { Resist. exp. media } \\
\left(\mathrm{kp} \mathrm{cm}^{2}\right)\end{array}$} & \multirow{2}{*}{$\begin{array}{c}\text { Coef. de paso } \\
(\mathrm{mS} / \mathrm{P} 3)\end{array}$} \\
\cline { 2 - 3 } & $\mathrm{mS}$ & $\mathrm{P} 3$ & \multirow{2}{*}{$\mathbf{0 , 7 2}$} \\
\hline Efectiva & 110,7 & 152,9 & \\
\hline Bruta & 59,2 & 82,2 & $\mathbf{0 , 6 6}$ \\
\hline Neta & 101,1 & 152,9 & $\mathbf{2}$ \\
\hline
\end{tabular}

\subsection{Muretes simples sometidos a carga excéntrica}

En este apartado se comparan los valores de muretes simples ensayados en seco (fase III), en relación a las previsiones teóricas para la sección transversal $\left(99 \times 14 \mathrm{~cm}^{2}\right)$, con huecos, de estos elementos.

Dado que la esbeltez 100/14 = 7,14 de los muretes es reducida, el análisis resistente que se realiza corresponde al de "agotamiento de una sección", sin incluir "efectos de segundo orden". Éstos empiezan a hacerse sentir para esbelteces superiores a 10 , según indican las diferentes normas y autores.

Las figuras 16 a 18 recogen los diagramas de interacción " $\mathrm{P} / \mathrm{P}_{\mathrm{o}}$ \# e/t", que proporcionan algunos de los diferentes análisis que se contemplan, y sobre ellos los valores obtenidos experimentalmente. En las tablas vecinas aparece la valoración numérica correspondiente. 
DIAGRAMAS DE INTERACCIÓN PARA "MURETES SIMPLES"

- Valores experimentales mS - SE - FASE III : (x)

- Propuestas de EC-6 y B.S-5628 :

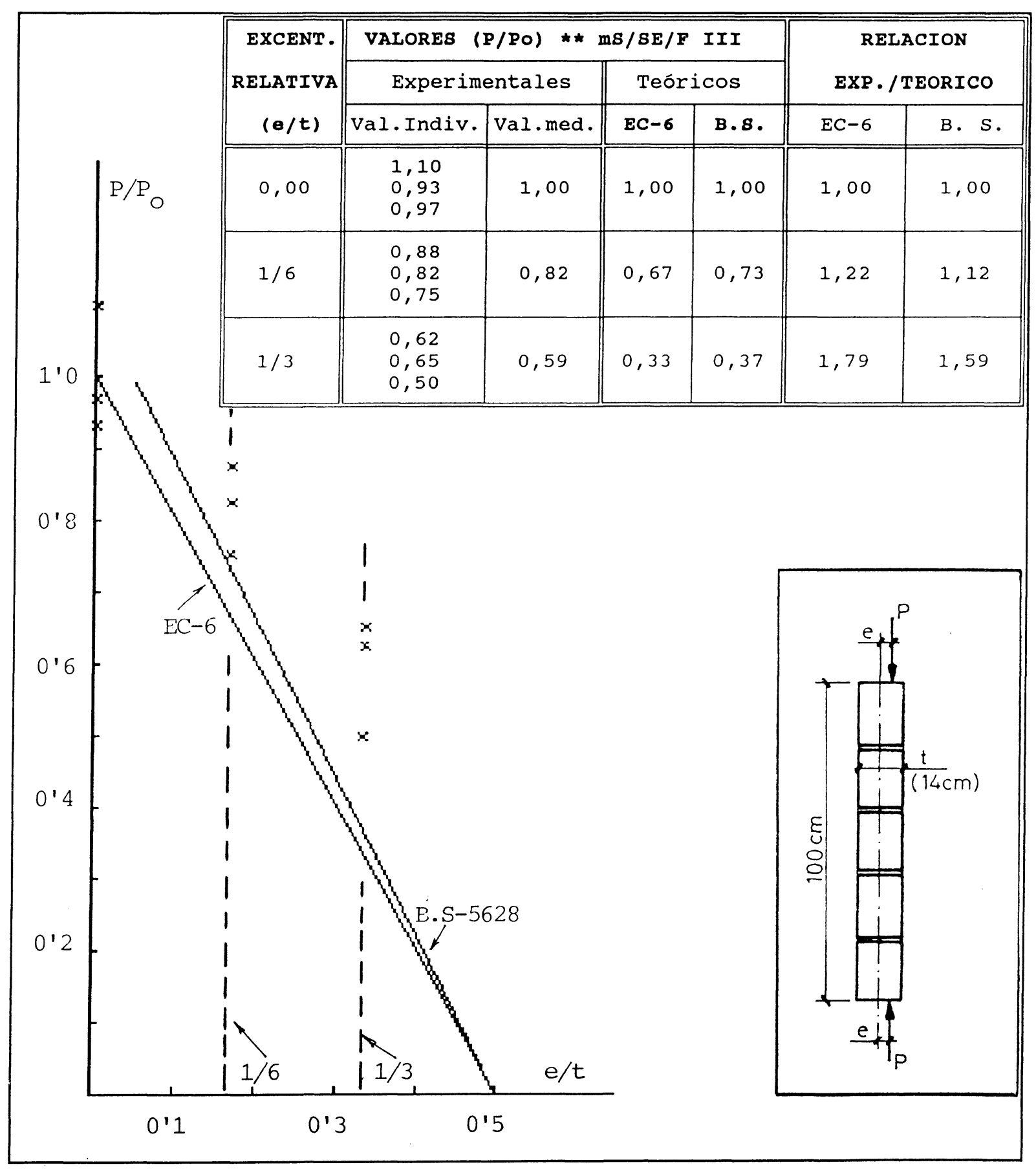

Fig. 16.

Valores $\left(\mathrm{P} / \mathrm{P}_{\mathrm{o}}\right)$, experimentales y teóricos para diferentes excentricidades relativas (e/t) de la carga aplicada a "MURETES SIMPLES" de $(1,0 \times 1,0) \mathrm{m}^{2}$ ensayados en seco (Carga centrada $\left.\mathrm{P}_{0}=82,1 \mathrm{Mp}\right)$. 
DIAGRAMAS DE INTERACCIÓN PARA "MURETES SIMPLES"

- Valores experimentales mS - SE - FASE III : (x)

- Propuestas de BUPSI

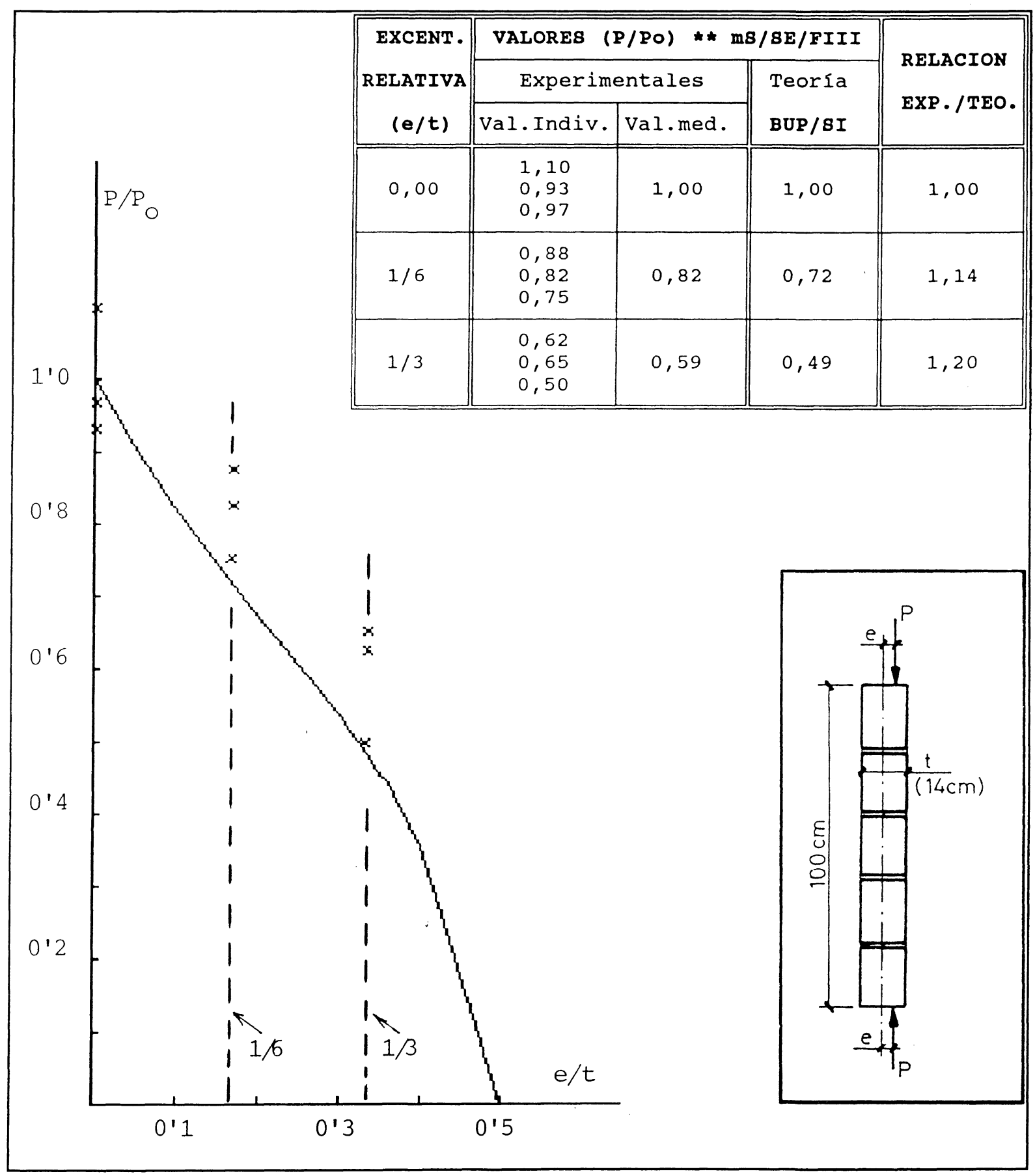

Fig. 17.

Valores $\left(\mathrm{P} / \mathrm{P}_{\mathrm{o}}\right)$, experimentales y teóricos para diferentes excentricidades relativas (e/t) de la carga aplicada a "MURETES SIMPLES" de $(1,0 \times 1,0) \mathrm{m}^{2}$ ensayados en seco (Carga centrada $\mathrm{P}_{0}=82,1 \mathrm{Mp}$ ). 
DIAGRAMAS DE INTERACCIÓN PARA "MURETES SIMPLES"

- Valores experimentales mS - SE - FASE III : (x)

- Propuestas de YOKEL ( $\mathrm{a}=$ variable $) \quad:(-)$

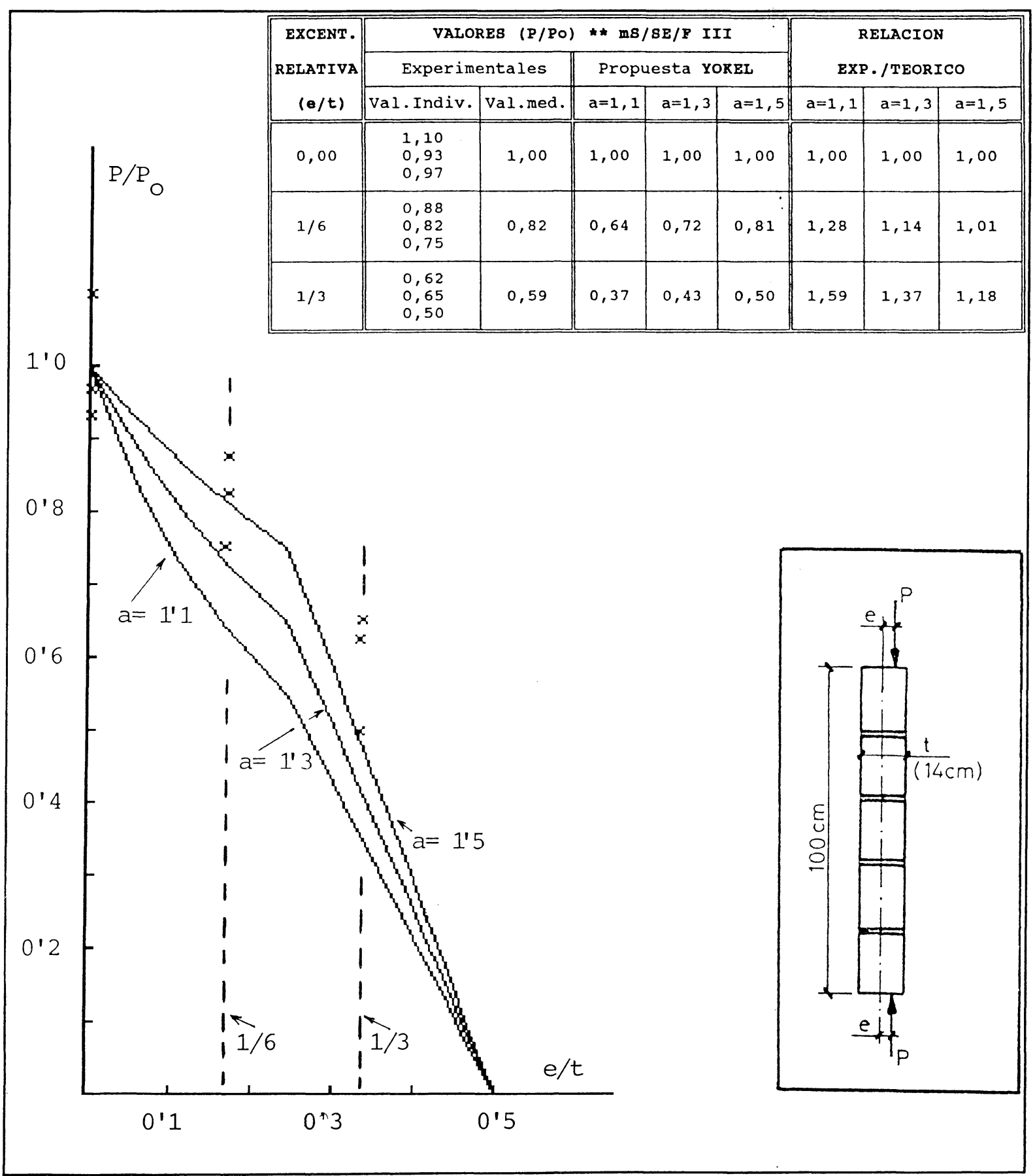

Fig. 18.

Valores ( $\mathrm{P} / \mathrm{P}$ ), experimentales y teóricos para diferentes excentricidades relativas (e/t) de la carga aplicada a "MURETES SIMPLES" de $(1,0 \times 1,0) \mathrm{m}^{2}$ ensayados en seco (Carga centrada $\mathrm{P}_{0}=82,1 \mathrm{Mp}$ ). 


\subsubsection{Valoración global del análisis de muretes} simples sometidos a cargas excéntricas

En la tabla 11 se presenta, para los muretes simples, una valoración global de la relación entre los valores experimentales de $\mathrm{P}_{\mathrm{u}} / \mathrm{P}_{\mathrm{o}}$ y los obtenidos de las propuestas desarrolladas, llegándose de la mencionada tabla a las siguientes conclusiones:

- Para la excentricidad de t/6, la evaluación de la carga resistente que proporcionan las diferentes teorías puede considerarse aceptable. Para el caso de la propuesta de $\operatorname{YOKEL}(a=1,5)$, la relación entre el valor experimental y teórico de $P_{\mathfrak{}} / P_{o}$ ha sido de 1,01 .

- Para la excentricidad de t/3, sólo algunas de las propuestas teóricas pueden considerarse adecuadas. Para el caso de la propuesta de YOKEL $(a=1,5)$, la relación entre el valor experimental y teórico de $P_{\imath} / P_{o}$ ha sido de 1,18 . Para este caso de excentricidades importantes, las propuestas de BS y EC-6 directamente aplicables a secciones macizas - son muy conservadoras.

Tabla 11

Relación entre las proporciones $\mathbf{P}_{\mathrm{u}} / \mathbf{P}_{\mathrm{o}}$ experimental y teórica en muretes simples secos (fase III)

\begin{tabular}{|c|c|c|c|c|c|c|c|}
\hline \multirow{2}{*}{\multicolumn{2}{|c|}{ Propuesta }} & \multicolumn{3}{|c|}{ EXC.e $=t / 6$} & \multicolumn{3}{|c|}{ EXC.e $=t / 3$} \\
\hline & & $\begin{array}{l}\text { Experi. } \\
\mathrm{P}_{\mathrm{u}} / \mathrm{P}_{\mathrm{O}}\end{array}$ & $\begin{array}{l}\text { Teórico } \\
\mathrm{P}_{\mathrm{u}} / \mathrm{P}_{0}\end{array}$ & $\begin{array}{c}\text { Relac. } \\
\text { Exp/Teo }\end{array}$ & $\begin{array}{l}\text { Experi. } \\
\mathrm{P}_{\mathrm{u}} / \mathrm{P}_{0}\end{array}$ & $\begin{array}{l}\text { Teórico } \\
\mathrm{P}_{u^{\prime}} \mathrm{P}_{0}\end{array}$ & $\begin{array}{l}\text { Relac. } \\
\text { Exp'Teo }\end{array}$ \\
\hline \multicolumn{2}{|c|}{ EC-6 } & 0,82 & 0,67 & 1,22 & 0,59 & 0,33 & 1,79 \\
\hline \multicolumn{2}{|l|}{ BS } & 0,82 & 0,73 & 1,12 & 0,59 & 0,37 & 1,59 \\
\hline \multicolumn{2}{|c|}{ BUPSI } & 0,82 & 0,72 & 1,14 & 0,59 & 0,49 & 1,20 \\
\hline \multirow{3}{*}{$\underset{2}{\stackrel{1}{0}}$} & $a=1,1$ & 0,82 & 0,64 & 1,28 & 0,59 & 0,37 & 1,59 \\
\hline & $a=1,3$ & 0,82 & 0,72 & 1,14 & 0,59 & 0,43 & 1,37 \\
\hline & $a=1,5$ & 0,82 & 0,81 & 1,01 & 0,59 & 0,50 & 1,18 \\
\hline \multicolumn{2}{|c|}{ PGCF } & 0,82 & 0,70 & 1,17 & 0,59 & 0,48 & 1,23 \\
\hline
\end{tabular}

\subsubsection{Diagramas de interacción dimensionales para} muretes simples, a partir de la resistencia a compresión de prismas simples. Comparación con los valores experimentales

Mediante el PGCF [12], y a partir de las resistencias a compresión del propio murete simple [f' $m=$ $\mathrm{f}(\mathrm{mS})$ ] y la de prismas simples de tres unidades [f'm $=\mathrm{f}(\mathrm{P} 3)]$, se han obtenido los diagramas de interacción de la sección transversal del murete simple de la figura 19. Puede observarse que cuando el dia-

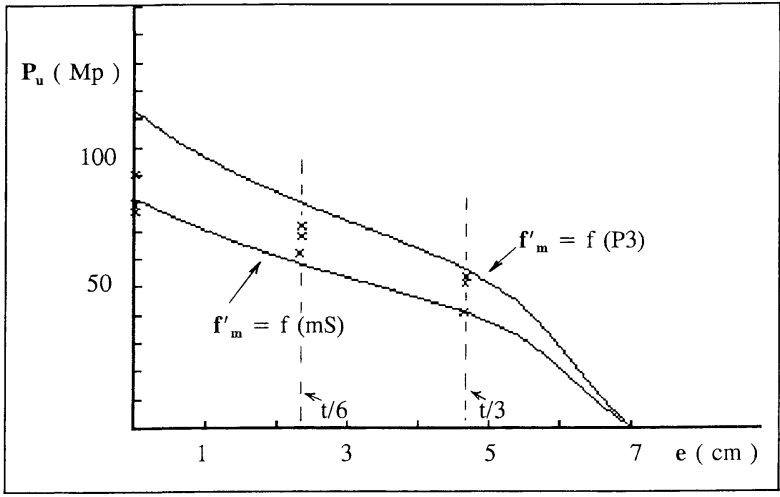

Fig. 19. Diagramas de interacción para muretes simples (mS), sobre su área efectiva, a partir de las resistencias a compresión: del propio murete $(\mathrm{mS})$, y la de prismas simples de tres unidades (P3).

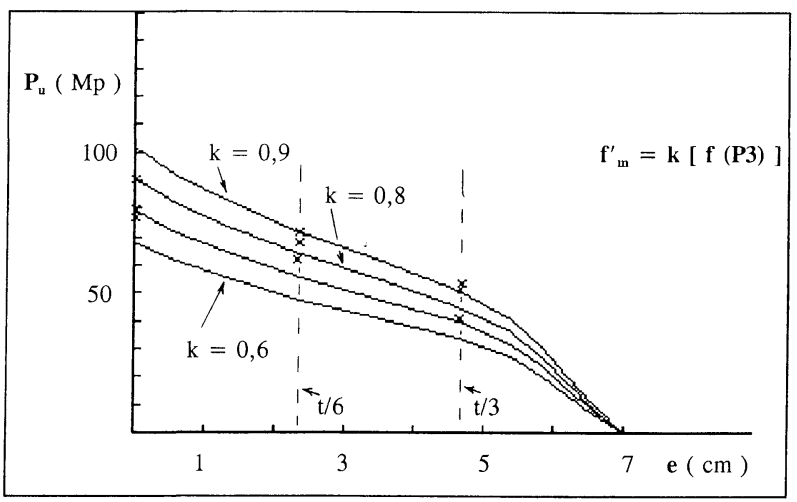

Fig. 20. Diagramas de interacción para muretes simples, sobre su área efectiva, a partir de las resistencias a compresión minorada, entre el 60 y el 90 por ciento, de prismas simples de tres unidades (P3).

grama es obtenido a partir de la resistencia de los prismas simples, los valores que se obtienen son mayores que las cargas experimentales de rotura del murete; siendo, por lo tanto, inseguro evaluarlos bajo este procedimiento.

\subsubsection{Coeficientes de paso "murete/prisma simples" para cargas excéntricas}

En la figura 20 se muestran diagramas de interacción para un murete simple, obtenidos a partir de minorar la resistencia a compresión de prismas simples de tres unidades; asimismo se indican las cargas excéntricas de rotura obtenidas experimentalmente en dichos muretes. La conclusión a la que se llega es la siguiente:

- El diagrama de interacción del murete simple, obtenido a partir del 80 por ciento de la resistencia a compresión de prismas simples de tres 
unidades, evaluados ambos sobre sus áreas efectivas, brindan valores ligeramente menores que sus cargas excéntricas de rotura experimentales. Por consiguiente, para cargas excéntricas, puede tomarse como coeficiente de paso "murete/prisma simples" el valor de 0,80 .

\subsection{Muretes armados sometidos a carga excéntrica}

5.3.1 Diagramas de interacción de muretes armados a partir de la resistencia a compresión de prismas rellenos

En [10] se demuestra que es aceptable tomar, como resistencia a compresión de los muretes de rellenos, el correspondiente a prismas rellenos de tres unidades. Es decir, un coeficiente de paso "murete/prismas rellenos" $=1$.

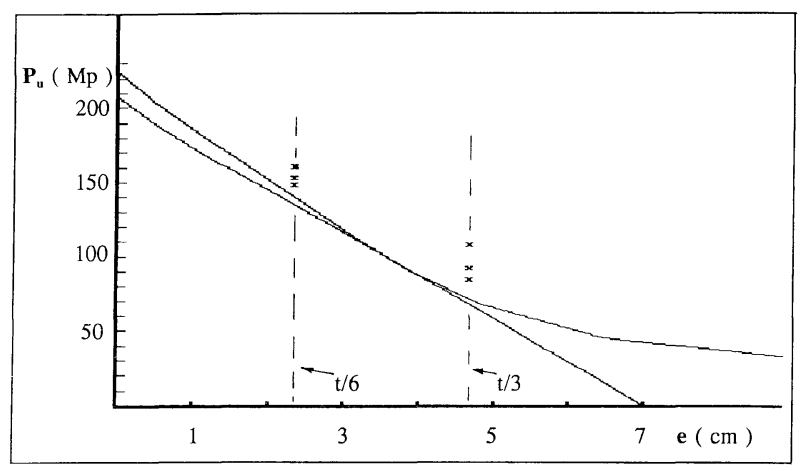

Fig. 21. Diagramas de interacción para muretes armados (1ø10@20 cm) a partir de la resistencia a compresión de prismas rellenos de tres unidades, con y sin considerar la contribución de la armadura vertical.

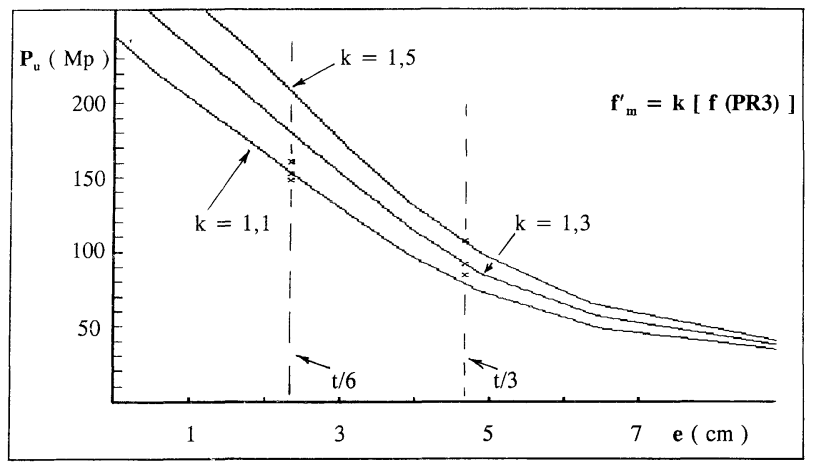

Fig. 22. Diagramas de interacción para muretes armados (1ø10@20 cm) obtenidos a partir de mayorar: entre el 10 y el 50 por ciento la resistencia a compresión de prismas rellenos de tres unidades.

En la figura 21 se han dibujado, a partir de la resistencia a compresión de prismas rellenos de tres unidades, los diagramas de interacción para muretes armados con y sin considerar la contribución de la armadura vertical, indicándose, asimismo, los valores experimentales de las cargas de rotura de los muretes. De dicha figura se llega a las siguientes conclusiones:

- En las excentricidades de t/6 y t/3 analizadas para la cuantía utilizada, es pequeña la contribución de la armadura vertical a resistir carga en dicha situación (entre un 4 y un 5 por ciento).

- Los valores experimentales de las cargas excéntricas de rotura son mayores, entre el 11 por ciento $(\mathrm{e}=\mathrm{t} / 6)$ y el 28 por ciento $(\mathrm{e}=\mathrm{t} / 3)$, que los obtenidos a partir del diagrama de interacción del murete armado (considerando la contribución de la armadura vertical).

Es, pues, aceptable y conservador evaluar las cargas de rotura de muretes armados, a partir de su diagrama de interacción, cuando se adopta como su resistencia a compresión la de prismas rellenos de tres unidades.

\subsubsection{Coeficiente de paso "murete armado/prisma relleno" para cargas excéntricas}

En la figura 22 se muestran diagramas de interacción obtenidos a partir de mayorar la resistencia a compresión de prismas rellenos de tres unidades indicándose, además, las cargas excéntricas de rotura obtenidas experimentalmente en muretes armados. La conclusión a la que se llega de dicha figura es la siguiente:

- Existe una buena aproximación (del 1 por ciento) entre las cargas experimentales excéntricas de rotura de muretes armados y los valores que brindan los diagramas de interacción obtenidos de mayorar: entre un 10 por ciento $(\mathrm{e}=\mathrm{t} / 6)$ y un 30 por ciento $(\mathrm{e}=\mathrm{t} / 3)$ la resistencia a compresión de prismas rellenos de tres unidades.

\subsection{Influencia del estado de saturación}

$\mathrm{Al}$ saturar los muretes, simples ( 48 horas) y armados (72 horas), la disminución en la carga media de

Tabla 12

Influencia de la saturación en muretes

\begin{tabular}{|l|c|c|c|}
\hline \multirow{2}{*}{$\begin{array}{l}\text { Muretes } \\
\text { ensayados }\end{array}$} & \multicolumn{3}{|c|}{$\begin{array}{c}\text { Relación entre las cargas de rotura: } \\
\text { saturado/seco }\end{array}$} \\
\cline { 2 - 4 } & $\mathbf{e}=\mathbf{0}$ & $\mathbf{e}=\mathbf{t} / \mathbf{6}$ & $\mathbf{e}=\mathbf{t} / \mathbf{3}$ \\
\hline Simples & 0,80 & 0,81 & 0,79 \\
\hline Armados & - & 0,91 & 0,85 \\
\hline
\end{tabular}


rotura es cuantificada en la tabla 12 . Puede observarse que la disminución porcentual en la carga de rotura por efecto de saturación ha sido: del 20 por ciento en muretes simples, y del 12 por ciento en muretes armados.

\section{Conclusiones}

En base a las propuestas de diversos investigadores y normativas analizadas, así como también de los resultados obtenidos experimentalmente en la presente investigación, pueden establecerse las siguientes conclusiones:

\subsection{Relativas a muretes simples}

\subsubsection{Muretes simples sometidos a carga uniformemente distribuida}

Los valores teóricos de resistencia a compresión para muretes simples (secos), a excepción de la propuesta del EC-6 [6] (un 10 por ciento mayor que el obtenido en los ensayos), guardan una buena relación con los valores obtenidos experimentalmente. Estos últimos son mayores a los de las normativas analizadas: en un 31 por ciento a la resistencia característica propuesta por $B S$ [3], y entre un 11 y un 12 por ciento a las resistencias medias (en sección efectiva) propuestas por $A C I$ [1] y NCMA [9], respectivamente.

El valor experimental del coeficiente de paso "murete/prisma simple", que relaciona la resistencia a compresión de ambos, es de 0,72 cuando se evalúa sobre sus áreas efectiva o bruta. Para todos los casos el prisma es de tres unidades.

\subsubsection{Muretes simples sometidos a cargas excéntricas}

Las cargas resistentes que proporcionan las diferentes teorías son aceptables para la excentricidad de t/6, y sólo algunas para la excentricidad de t/3. Si se analizan aquellas que se aproximan más a los valores experimentales, se tiene que:
- En los muretes simples sometidos a cargas excéntricas, los valores experimentales de las relaciones $\mathrm{P}_{\mathrm{u}} / \mathrm{P}_{\mathrm{O}}$ fueron mayores a los teóricos: entre el 1 por ciento (YOKEL: $\mathrm{a}=1,5$ ) y el 12 por ciento (BS) a la excentricidad de $\mathrm{t} / 6$; y entre el 18 por ciento (YOKEL: $\mathrm{a}=1,5$ ) y el 20 por ciento (BUPSI) a la excentricidad de $\mathrm{t} / 3$.

- El diagrama de interacción del área efectiva del murete simple, que mejor se aproxima a sus cargas excéntricas de rotura, es el que se obtiene a partir del 80 por 100 de la resistencia a compresión de los prismas simples de tres unidades.

\subsection{Relativas a muretes armados sometidos a cargas excéntricas}

Las cargas resistentes que proporciona el diagrama de interacción de la sección del murete armado, obtenido a partir de la resistencia a comprensión de prismas rellenos de tres unidades - mediante el PGCF [12]-, es aceptable y conservador. Los valores experimentales de las cargas excéntricas de rotura en muretes armados son mayores: entre el 11 por ciento $(\mathrm{e}=\mathrm{t} / 6)$ y el 28 por ciento $(\mathrm{e}=\mathrm{t} / 3)$, que los obtenidos a partir del diagrama de interacción de su sección (considerando la contribución de la armadura vertical).

El diagrama de interacción del murete armado, que mejor se aproxima a sus cargas excéntricas de rotura, es el que se obtiene mayorando la resistencia a compresión de los prismas rellenos de tres unidades en: un 10 por ciento para e $=\mathrm{t} / 6$, y un 30 por ciento para $\mathrm{e}=\mathrm{t} / 3$.

\subsection{Relativas a la influencia de la saturación en las cargas de rotura de muretes}

La disminución porcentual en las cargas de rotura, por efecto de saturación, ha sido: del 20 por ciento en muretes simples y del 12 por ciento en muretes armados.

\section{BIBLIOGRAFÍA}

[1] ACI-531 (Rev. 1983). Building Code Requirements for Concrete Masonry Structures, American Concrete Institute, 1983.

[2] ASTM E447-84. Standard Test Methods for Compressive Strength of Masonry Prisms, 1984.
[3] BS 5628 (Part 1). Code of Practice for Use of Masonry: Structural Use of Unreinforced Masonry, 1978.

[4] BS 5628 (Part 2). Code of Practice for Use Masonry: Structural Use of Reinforced and Prestressed Masonry, 1985 
[5] CEN/TC 125 N63. Draft European Standard - MasonryMethods of Test, 1990.

[6] EUROCODE EC6 (Part 1). Common Unified Rules for Unreinforced Masonry Structures, Commission of the European Communities, Brussels, 1988.

[7] EUROCODE EC6 (Part 2). Common Unified Rules for Reinforced Masonry Structures. Commission of the European Communities. Brussels, 1989

[8] Instituto EDUARDO TORROJA. PIET-70: Obras de Fábrica. Prescripciones del Instituto Eduardo Torroja. Madrid, 1971.

[9] NCMA. National Concrete Masonry Association. Specification for the Design and Construction of Load-bearing Concrete Masonry, P.O. BOX 781, Herndon. Virginia 22070, USA, 1987.
[10] TUESTA, N. F.: "Estudio teórico-experimental de la resistencia de Estructuras de Fábrica de Bloques de Hormigón, con o sin armaduras, sometidas a flexo-compresión transversal a su plano". Tesis Doctoral ETSICCP de Santander. Universidad de Cantabria, 1992.

[11] UNE 41-172-89. BLOQUES DE HORMIGON: Método de Ensayo pura Determinar la Resistencia a compresión. Asociación Española de Normalización (AENOR), 1989.

[12] VILLEGAS, L. "Análisis en teoría de segundo Orden de Pilas Altas de Viaductos. Aplicación al Proyecto de Pilas de Puentes Construidos por Avance en Voladizo". Tesis Doctoral ETSICCP de la Universidad de Cantabria, 1981.

[13] YOKEL, F. Y.; MATHEY, R. G. \& DIKKERS, R. S. Strength of Masonry Walls Under Compressive and Transverse Loads, US Nat. Bur. Stand. (Bldg. Sci. Ser 34). March, 1971.

\section{JORNADAS SOBRE CEMENTO ALUMINOSO}

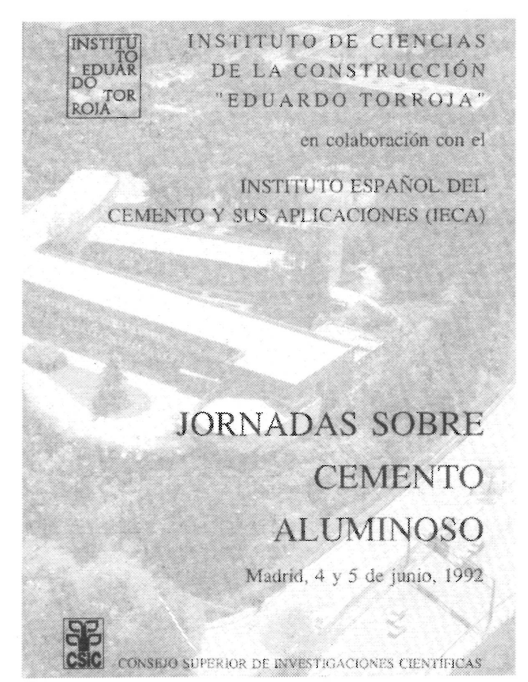

(c) Consejo Superior de Investigaciones Científicas Licencia Creative Commons 3.0 España (by-nc)
El Instituto Eduardo Torroja publica íntegramente -en colaboración con el Instituto Español del Cemento y sus Aplicaciones (IECA) - Ias "Jornadas sobre cemento aluminoso", celebradas en junio de 1992.

Se recogen las conferencias dictadas por destacados especialistas en la materia, así como las intervenciones suscitadas en los coloquios y en la interesante Mesa Redonda, tras la cual se clausuraron las Jornadas.

El tratamiento dado al tema del hormigón y cemento aluminoso por los intervinientes abarca tanto aspectos rigurosos científico-técnicos como de índole práctica, abordando temas de corrosión de armaduras, durabilidad, protección y reparación de los elementos constructivos con daños causados por diversas motivaciones ampliamente expuestas.

Este libro será, sin duda, una eficaz ayuda tanto para el científico o el técnico estudioso de esta materia, como para aquellos profesionales interesados en un tema de tanta importancia y actualidad como es el relacionado con los hormigones de cemento aluminoso.

Un volumen de 15,5 ×22,5 cm, 186 págs., con figuras de línea, gráficos, tablas, etc. 